\title{
The HBT effect: Trading zones in twentieth century physics
}

\author{
Indianara Silva*1,20 \\ ${ }^{1}$ Universidade Estadual de Feira de Santana, Departamento de Física, BA, Brasil \\ ${ }^{2}$ Universidade Federal da Bahia, Departamento de Educação, Programa de Pós-Graduação em Ensino, Filosofia e História das \\ Ciências, BA, Brasil
}

Received on February 24, 2019; Revised on April 10, 2019; Accepted on June 15, 2019.

\begin{abstract}
In 1956, Robert Hanbury Brown and Richard Twiss (HBT) reached an unexpected experimental result that caused a heated debate in the physics community. Contrary to Einstein's quantum of light, they asserted that they had separately detected two photons at the same time. As one may imagine, other physicists challenged this experimental result, noting that if it were correct, quantum theory would need to be reformulated. Once the controversy began, physicists from different subcultures of physics had to create a trading zone capable of making communication possible. As a result, both theoretical and experimental traditions developed significantly during the HBT debate. This paper illustrates how Galison's trading zone aids our understanding of the communication used to discuss the HBT results.
\end{abstract}

Keywords: Robert Hanbury Brown, Richard Quentin Twiss, Edward Purcell, Eric Brannen, Harry I. S. Ferguson, The HBT Effect, The HBT Controversy, Trading Zone, Scientific Practice, History of Science, History of Physics.

\section{Introduction}

In the 1950s, Robert Hanbury Brown and Richard Quentin Twiss performed an experiment that challenged the canonical concept of the photon. In the Hanbury Brown-Twiss (HBT) experimental set up, a half-silvered mirror split a low-intensity light source into two components and two photomultipliers then detected these components separately. Due to the type of source used, one would expect only single-photons to arrive at the mirror. Consequently, given the canonical concept of the photon (a small, invisible and localized particle) established by the old quantum theory, one would not expect systematic coincidences. Surprisingly, the HBT experiment demonstrated the contrary. It indeed detected a significant correlation between photons, i.e., photons arriving simultaneously in two detectors. If a single-photon cannot split in two, how was it that they found a correlation between photons? Either they, or quantum theory, were wrong. Unsurprisingly, a heated debate in the scientific community followed.

The early controversy surrounding the HBT experimental results, which seemed to contradict quantum theory predictions, revealed how scientists from different subcultures of physics created a line of communication in order to understand what was at stake in the HBT correlation. How did they enable this communication? To answer this question, we will consider the notion of the trading zone.

*Correspondence email address: isilva@uefs.br
Applying anthropological linguistics, the historian and philosopher of science Peter Galison argues that a trade between cultures (e.g. engineering, experimental physics and theoretical physics) is possible using an analogy creole and pidgin languages. Creole is a native language and each subculture of physics has its own, while pidgin is the contact language that enables different groups of physicists to communicate with each other $[1,2,3$, $4,5]$. Theorists, experimenters, and instrument makers with a range of training and backgrounds can create a language set that enables them to collaborate. In doing so, they "establish[ed] a place where ideas, data, and equipment could be passed back and forth between groups - constituting a trading zone" [1, p. 817].

From this perspective, Galison suggests thinking of "science as an intercalated field of subcultures" [2, p. 25], and consequently criticizes the positivist view of science as unity, as well as the antipositivist view of "theory and experiments as inextricably linked" [6, p. 841]. Interaction between subcultures thus occurs through a trading zone - "a social, material, and intellectual mortar binding together the disunified traditions of experimenting, theorizing, and instrument building" [1, p. 803]. In their interactions, different communities therefore ensure that science advances. In his book Image and Logic, Galison notes that the Nobel Prize winning American physicist Julian Schwinger created a pidgin language to establish a trading zone between field theory and electrical engineering during WWII. Schwinger and engineers were 
therefore able to share a common language and thus collaborate on microwave radiation [1].

Here we use the notion of trading zone to demonstrate how scientists from different physical science traditions created a line of communication in order to understand the 1956 HBT experimental results, using primary and secondary sources. The establishment of what it is widely known as the HBT effect - the bunching of photons - as a phenomenon involved interactions between scientists from distinct subcultures of physics. The first section briefly presents the HBT experimental results, the second the HBT trading zones, while the article finishes with some concluding remarks.

\section{The HBT Experiment}

In 1956, Hanbury Brown and Twiss published an experimental result that "put the cat among the pigeons" [7, p. 120], thus causing a heated debate in the physics community [8]. The HBT experiment was a laboratory test to verify whether the principle used to detect the angular diameter of radio stars could be applied to visible stars. Their hypothesis was that "the time of arrival of photons at the two photocathodes should be correlated when the light beams incident upon the two mirrors are coherent" $[9$, p. 27]. To test this, they used a mercury arc as a low intensity light source, a system of filters to select wavelengths, a half-silvered mirror, two photomultipliers to detect the light beams, and a correlator to compare the two signals (Fig. 1) [8, 9].

For the HBT theoretical model, they applied classical electromagnetic wave theory and the quantum theory to the detection process, in order to calculate the correlation between the two light beams. Their expression depended on the quantum efficiency of the detectors, the number of quanta incident on the detector and excess noise $[8$,
9]. Comparing the HBT theory with their experimental results, Hanbury Brown and Twiss confirmed their hypothesis and concluded that "the experiment shows beyond question that the photons in two coherent beams of light are correlated, and this correlation is preserved in the process of photoelectric emission" [9, p. 29]. What did the HBT result mean? Since they used a low intensity source, one would expect a single photon to arrive at the half-silvered mirror and, consequently, no systematic coincidences should be detected. Clearly, what HBT found in 1956 brought Einstein's 1905 quantum of light into question, since they simultaneously detected two photons [10].

\section{The HBT Trading Zones}

How can the notion of a trading zone help us understand the early HBT debate? How did these collaborations come? Let us start with Hanbury Brown and Twiss. The former earned a bachelor degree in electrical engineering and a doctorate in radio astronomy, while the latter completed the Mathematical Tripos and a doctorate in science. Before they worked together, both had worked on radar during WWII [8]. One of the most significant lessons from wartime was how fruitful and successful a research program could be when both theorists and experimenters came together to solve a specific problem. This was the case with the atomic bomb and radar - the products of scientific interaction between theorists, experimenters, engineers, and instrument makers [2]. Hanbury Brown and Twiss had certainly learned from this lesson.

Their collaboration began before the 1956 HBT paper, when they worked on a radio intensity interferometer. When Hanbury Brown decided to construct this, he had envisioned how to proceed in terms of experimental apparatus. "Hanbury [Brown] was a superb and imaginative

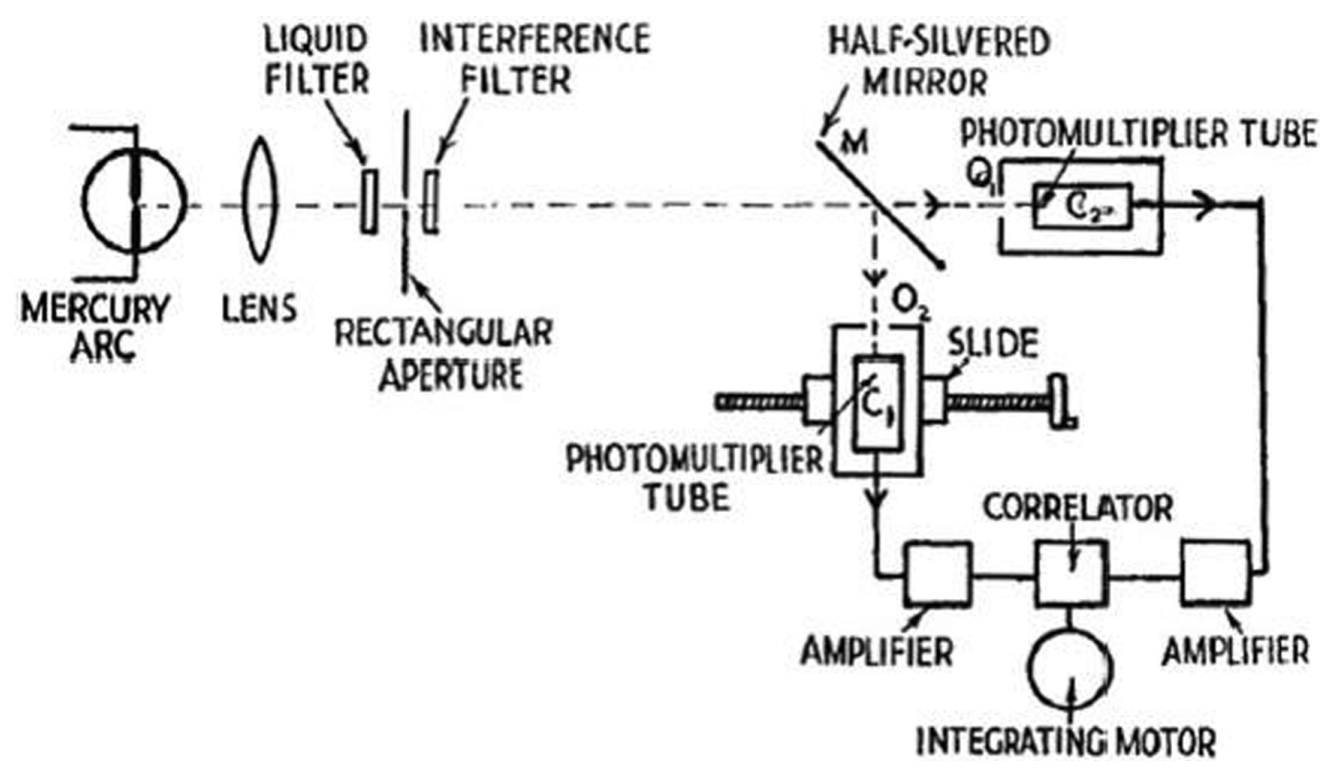

Figure 1: The HBT experimental set up. Source: [9, p. 28]. 
engineer, a natural astronomer, and a true visionary" [11]. Though he knew that he had the skills to build this new type of interferometer, Hanbury Brown needed to know if it would be sensitive enough to measure the angular diameter of radio stars. He could have read up on the underlying mathematics of this construction. Instead, acknowledging the importance of scientific collaboration, Hanbury Brown started looking for a theorist who could work on his interferometer idea. He knew his limitations regarding mathematics, and the new instrument would require a very sophisticated mathematical approach. As Hanbury Brown later recalled, "unfortunately I didn't know enough mathematics to work out the answer, so I asked my old friend Vivian Bowden, who seemed to know everyone, to find me someone who did." He continued, "Vivian found me someone called Richard Twiss who, like me, was born in India of an Army family, but, unlike me, was a talented mathematician" [7, p. 105].

While Hanbury Brown and his graduate students were working on designing the new interferometer, Twiss worked on the mathematics of the new apparatus. Since they were working in different English cities, Hanbury Brown in Manchester and Twiss in Baldock, they created a scientific network. To maintain this collaboration, Twiss often visited Manchester and was in constant correspondence with Hanbury Brown. In this way, they were able to establish a trading zone - a site in which engineers, experimenters, and theorists, despite being from different subcultures, were able to collaborate $[2 ; 4]$. Although each subculture has its own idiosyncrasies and way of operating, a place was created in which scientists could construct a common culture and share consensual practices, values and ideas. "Cultures in contact can coordinate their actions and beliefs - even in the absence of shared global views about meaning" [12, p. 16]. By creating a zone of exchange, Hanbury Brown and Twiss worked together to construct the intensity interferometer - Hanbury Brown constructing the apparatus and Twiss developing the theory. Each contributed to the other's subculture - the traditions of experimenting and theorizing - without losing their distinct identities and practices. Following this collaboration, Hanbury Brown did not become a theorist, nor did Twiss become an experimenter. Conversely, they did know how to exploit each other's skills, ideas, inventions, and knowledge to construct the new interferometer.

The success of the intensity interferometer, which functioned properly in a turbulent medium, motivated Hanbury Brown and Twiss to take a further step. Using the same theoretical principles and slightly adapting the experimental apparatus, they decided to construct an interferometer capable of working at visible wavelengths. However, before The Narrabri Stellar Intensity Interferometer (NSII) ${ }^{1}$ became a reality, they conducted the HBT laboratory test, which caused a great deal of trouble.

\footnotetext{
1 The NSII is located in New South Wales, Australia.
}

During the HBT controversy, experimenters and theorists around the world established a new trading zone to try to understand and interpret the HBT correlation. Let us focus on the early debate between Edward Purcell, Eric Brannen, and Hanbury Brown and Twiss. Purcell, the 1952 Nobel Prize winning American physicist, worked on the magnetic resonance of nuclei and radio astronomy [13], while Brannen was a Canadian physicist working in microtron research [14]. Their specific expertise resulted in different interpretations of the HBT results. While Brannen and his graduate student Harry I. S. Ferguson claimed that if the HBT coincidence were correct, "it would call for a major revision of some fundamental concepts in quantum mechanics" [15, p. 482], Purcell asserted that it was an elementary effect of physics.

Brannen and Ferguson decided to perform an experiment a la HBT to verify whether they would attain the same experimental result. Indeed, they did not find any coincidences between photons - the quantum theory survived [15]. Even before the HBT 1956 experiment, the Hungarian physicist Lajos Jánossy and his research group experimentally verified this quantum theory prediction: "the pulses recorded by the two multipliers are independent of each other and thus no systematic coincidences are to be expected" [16, p. 57]. Two different pieces of evidence against one made it seem as if the HBT correlation was wrong.

Contrary to Brannen and Ferguson, Purcell pointed out that "the Brown-Twiss effect, far from requiring a revision of quantum mechanics, is an instructive illustration of its elementary principles" [17, p. 1449-50]. He published a new means of interpreting the HBT correlation using a language different from that of Hanbury Brown and Twiss. Purcell used a pidgin language to communicate with the actors involved in the controversy and the physics community. That is, a contact language used to enable communication between two different groups or communities when they need to trade or exchange [2]. Purcell thus established a zone of exchange between the communities - experimental and theoretical physicists, astronomers and mathematicians - creating a meeting point where they could understand and discuss the HBT experiment in a different way. As he said, "There is nothing in the argument [...] that is not implicit in the discussion of Brown and Twiss, but perhaps I may clarify matters by taking a different approach" [17, p. 1449]. Beautifully linking the language of quantum statistics to the language of coincidence experiments, Purcell now calculated the HBT correlation as depending on the number of counts, observation time and correlation time.

Purcell's 1956 paper did not end the debate. Brannen, Purcell, Hanbury Brown and Twiss exchanged a great deal of correspondence and created a wider trading zone. Brannen and Ferguson, for example, did not agree with the theoretical language Hanbury Brown and Twiss used. As Brannen wrote to Purcell, "he [Hanbury Brown] offered no suggestion of "photon overlap" or in- 
deed any clarification of the physical principles involved but only stated that he thought their experiments were more accurate" [18]. It seems that, if HBT had used photon overlap language, Brannen and Ferguson would have softened their approach. Purcell replied, saying that "talking about interference of photons is the easiest way to go astray in such matters" and continued:

The electromagnetic field is a classical field after all, which is why the Brown-Twiss effect only appears odd if one looks at it from a particle point of view; its oddness being simply the peculiarity of bosons. One might turn it around and say that this is a nice example of the fact that a classical field must have bosons for its quanta [19].

What Purcell did was to create a trading zone where Brannen, Hanbury Brown and himself could come together to communicate using a pidgin language, although they explained the same phenomenon in different ways. While Brannen was now suggesting the consideration of photons as a wave-packet, Purcell treated photons as bosons, and Hanbury Brown and Twiss preferred to approach light as a classical electromagnetic field. Each subculture of physics has its own way of representing light, which does not have equal meaning for an experimenter, a theorist, or an engineer. "Interpretations could conflict, or could come to consensus", as happened in the HBT debate, "but this intermediate set of linguistic and procedural practices bound experiments, instrument makers, and theorists in collaboration" [2, p. 805]. The established trading zone contributed to the learning of new concepts, languages and techniques. As Purcell said to Brannen, "I must say I have learned some physics in the course of it, which makes me grateful for the stimulation provided by the intrepid experimenters, yourselves included, who have gone back to really fundamental experiments" [19]. Collaborating with Purcell, even at a distance, also improved Twiss' theoretical practice. He once wrote to Purcell, "The reason for my writing you is not very serious, but I cannot get the same answer as you do in deriving the "coherence length" for light with a rectangular bandwidth" [20].

Purcell was therefore a central figure, who created a language set in the trading zone that helped two different groups communicate. He was the first physicist to acknowledge the HBT correlation as an effect in physics, contributing to the later creation of a creole language the product of exchange - "a pidgin extended and complexified to the point where it can serve as a reasonably stable native language" [2, p. 831-32]. The HBT correlation is widely known as the HBT Effect - the bunching of photons- as a characteristic of a boson system $[8,21$, $22]$.

\section{Concluding Remarks}

We have seen that, even when they are from different scientific traditions, scientists can collaborate by creating a trading zone in which they can share certain actions, values and beliefs. By trying to create a common language to communicate with each other, our protagonists - from distinct subcultures of the physical sciences and astronomy - strengthened the wider culture of science, including the development of the fields of astronomy and quantum optics.

\section{References}

[1] P. Galison, Image and Logic (University of Chicago Press, Chicago, 1997).

[2] P. Galison, in: The disunity of science: Boundaries, contexts, and power, edited by P. Galison and D.J. Stump (Stanford University Press, Stanford, 1996).

[3] P. Galison, in: Trading zones and interactional expertise: Creating new kinds of collaboration, edited by M.E. Gorman (The MIT Press, Cambridge, 2010).

[4] P. Galison, in: The science studies reader, edited by M. Biagioli (Psychology Press, London, 1999).

[5] P. Galison, in: Science in the Twentieth Century, edited by J. Krige and D. Pestre (Hardwood, Amsterdam, 1997).

[6] P.O. Long, Isis 106, 840 (2015).

[7] R.H. Brown, Boffin: a personal story of the early days of radar, radio astronomy and quantum optics (CRC Press, London, 1991).

[8] I. Silva and O. Freire Jr, Historical Studies in the Natural Sciences 43, 453 (2013).

[9] R. H. Brown and R.Q. Twiss, Nature 177, 4497 (1956).

[10] A. Einstein, Annalen der Physik 17, 132 (1905).

[11] B. Lovell and R.M. May, Nature 416, 6876 (2002).

[12] P. Galison, in: Scientific practice: Theories and stories of doing physics, edited by J. Z. Buchwald (The University of Chicago Press, Chicago, 1995).

[13] R.V. Pound, Edward Mills Purcell, available in https: //www.nap.edu/read/9977/chapter/12, accessed on $25 / 01 / 2019$.

[14] D. R. Moorcroft, A History of the Department of Physics and Astronomy: The University of Western Ontario, available in http://www.physics.uwo.ca/ drm/ history/dept_history/history_pic.htm, accessed on 25/01/2019.

[15] E. Brannen and H.I.S. Ferguson, Nature 178, 4531 (1956).

[16] A. Ádám, L. Jánossy, and P. Varga, Acta Phys. Hungary 4, 301 (1955).

[17] E.M. Purcell, Nature 178, 4548 (1956).

[18] E. Brannen and H. I. Ferguson to E. M. Purcell (Harvard University Archives, Cambridge, 1956), correspondence.

[19] E. M. Purcell to E. Brannen (Harvard University Archives, Cambridge, 1956), correspondence.

[20] R. Q. Twiss to E. M. Purcell (Harvard University Archives, Cambridge, 1956), correspondence.

[21] I. Silva and O. Freire Jr, Historical Studies in the Natural Sciences 45, 511 (2015).

[22] K. Hentschel, Photons: The History and Mental Models of Light Quanta (Springer, Switzerland, 2018). 